\title{
Ocular Volumetry Using Fast High-Resolution MRI during Visual Fixation
}

\author{
K. Tanitame, T. Sone, T. Miyoshi, N. Tanitame, K. Otani, Y. Akiyama, M. Takasu, S. Date, Y. Kiuchi, and K. Awai
}

\begin{abstract}
BACKGROUND AND PURPOSE: Volumetry may be useful for evaluating treatment response and prognosis of intraocular lesions. Phantom, volunteer, and patient studies were performed to determine whether ocular MR volumetry is reproducible.

MATERIALS AND METHODS: Half-Fourier single-shot RARE and FSPGR sequences at 1.5T with a 76-mm-diameter surface coil were optimized to obtain still ocular images. Volumetry accuracies of each sequence were compared with simulated subretinal phantom volumes. Ocular volumetry was performed in 15 volunteers twice in 1 week by using contiguous axial images of the globes while the subjects stared at a target, and images were acquired in 2 seconds before the subjects were instructed to blink, with this process repeated as necessary. Imaging, intraobserver, and interobserver reproducibility for volumes of the whole eyeball and anterior chamber were assessed. Ocular volumetry was also performed in 6 patients with intraocular tumors before and after treatment.
\end{abstract}

RESULTS: The phantom study demonstrated that measurement error rates with RARE were significantly lower than with FSPGR ( $P<.01)$. The volunteer study demonstrated excellent imaging and intraobserver reproducibility of RARE volumetry for whole eyeballs and anterior chambers $(P<.01)$. Although no interobserver differences were observed in anterior chamber volume measurement $(P=.33)$, there was a significant difference between the 2 observers in eyeball volume measurement $(P<.01)$. Follow-up volumetric data were useful for treatment decisions in all patients.

CONCLUSIONS: Ocular volumetry from contiguous ultrafast RARE images obtained during visual fixation is feasible in volunteer and patient studies and is superior to FSPGR images.

ABBREVIATIONS: FSPGR = fast-spoiled gradient recalled-echo; Gd = gadolinium; Gd-DTPA = gadolinium-diethylene-triamine pentaacetic acid; OCT = optical coherence tomography; RARE = rapid acquisition with relaxation enhancement

$\mathbf{M}_{\mathrm{m}}^{\circ}$ otion artifacts due to voluntary or involuntary eye movement present a problem for ocular imaging. Motion artifacts are clinically addressed with real-time imaging methods such as sonography ${ }^{1-3}$ and OCT, ${ }^{4-6}$ but the diagnostic performance of sonography depends on the skill of the examiner, and sonography cannot reach the posterior segment in hyphemic eyes. While OCT provides ocular images with high temporal and spatial resolution, it cannot image the whole eyeball or large intraocular lesions.

Received April 6, 2012; accepted after revision July 14.

From the Departments of Diagnostic Radiology (K.T., S.D., M.T., K.A.) and Ophthalmology and Visual Science (T.S., Y.K.), Graduate School of Biomedical Sciences, and Department of Environmetrics and Biometrics Research Institute for Radiation Biology and Medicine (K.O.), Hiroshima University, Hiroshima, Japan; Department of Clinical Radiology (T.M., Y.A.), Hiroshima University Hospital, Hiroshima, Japan; and Department of Radiology (N.T.), Hiroshima City Asa Hospital, Hiroshima, Japan.

Please address correspondence to Keizo Tanitame, MD, Department of Diagnostic Radiology, Graduate School of Biomedical Sciences, Hiroshima University, Hiroshima, Japan, 1-2-3 Kasumi, Minami-ku, Hiroshima 734-8553, Japan;

e-mail:tntrad@ms1.megaegg.ne.jp

http://dx.doi.org/10.3174/ajnr.A3305
CT is useful for emergency diagnosis of eye trauma ${ }^{7,8}$ and detection of intraocular calcifications. ${ }^{9} \mathrm{CT}$ exposes the eyeball to radiation, however, and yields lower contrast images of the ocular anatomy than MR imaging. MR imaging by using 2D FSE sequences, 3D FSE sequences, and 3D gradient-recalled-echo sequences can provide images of the eyeball with good contrast. ${ }^{10-12}$ However, MR imaging by using these sequences has lower temporal resolution than other ocular imaging methods and is often degraded by motion artifacts due to eye movement.

2D fast MR images such as half-Fourier single-shot RARE, balanced steady-state free precession, and FSPGR have the advantage of higher temporal resolution than conventional 2D FSE sequences. ${ }^{13-16}$ Optimized half-Fourier single-shot RARE and FSPGR sequences may provide ocular T2- and T1-weighted images, respectively, with fewer motion artifacts than the conventional images. Also, MR imaging scans with the subject staring at a target can yield a reproducible still image of the eye, ${ }^{17}$ and small surface receiver coils can provide ocular images with high spatial resolution. ${ }^{18-20}$ These methods may be combined to produce fast 


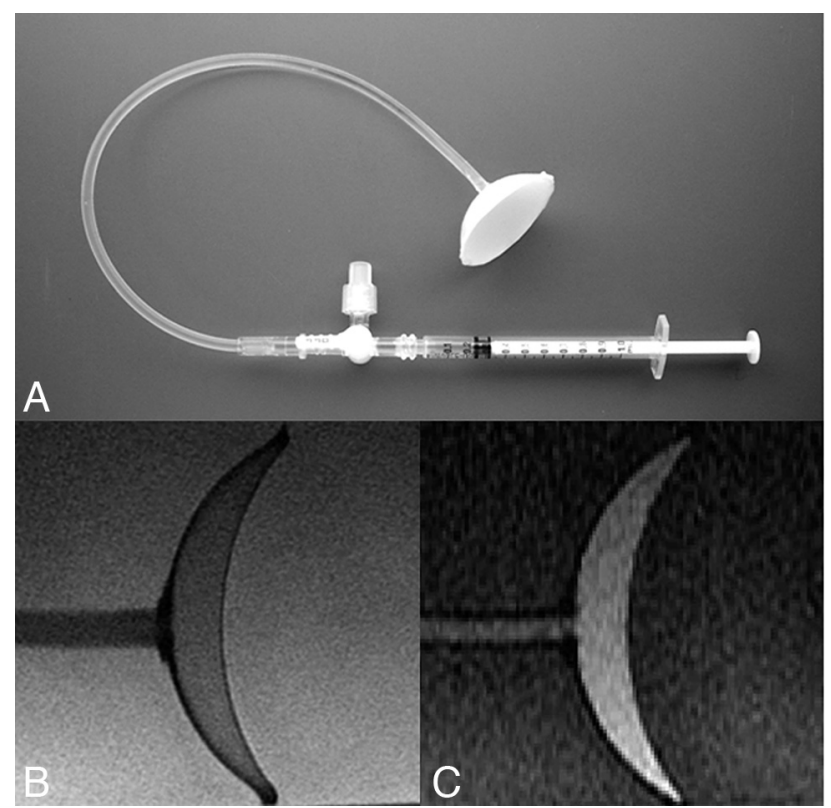

FIG 1. A, Retinal detachment phantom for evaluating the accuracy of ocular MR volumetry. This phantom consisted of a cut 38-mm-diameter table tennis ball and a rubber film. The film was placed on the cut end of the ball, a small hole was made in the posterior pole of the ball, and a tube was connected to the hole. To have the phantom simulate several types of retinal detachment, we injected different volumes of a $0.5-\mathrm{mmol} / \mathrm{L}$ Gd-DTPA solution from a small syringe via the tube connected to the hole. Representative half-Fourier single-shot RARE $(B)$ and enlarged (X1.6) FSPGR $(C)$ images of the retinal detachment phantom injected with $2.00 \mathrm{~mL}$ of a solution containing Gd-DTPA are shown.

high-resolution MR images for morphologic diagnosis of ocular lesions.

Because volumetric evaluation of the eyeball can be useful for follow-up of intraocular tumors, ${ }^{21}$ we assessed the feasibility of ocular volumetry by using fast MR images, a small surface coil, and the fixation of visual focus. The accuracy of volumetry was compared by using 2 different imaging sequences (half-Fourier single-shot RARE and FSPGR) for a phantom study, and the reproducibility of ocular volumetry was evaluated in healthy volunteers by using the RARE sequence and measuring anterior chamber volume and whole-eye volume. This volumetric method was also applied to a small group of patients with intraocular tumors.

\section{MATERIALS AND METHODS Phantom Model}

To evaluate the accuracy of ocular MR volumetry, we devised a retinal detachment phantom consisting of a cut table tennis ball that was designed to represent a part of the eyeball and a rubber film designed to represent the retinal membrane (Fig 1A). Then, to measure the subretinal volumes, we injected a volume of 1.00$4.00 \mathrm{~mL}$ of a solution containing $0.5 \mathrm{mmol} / \mathrm{L}$ of gadolinium (in the form of Gd-DTPA) as artificial blood into the simulated subretinal space of the phantom.

\section{Subjects}

The present study received institutional review board approval, and informed consent was obtained from all participants before entry into the study. Fifteen healthy volunteers were recruited from the staff at Hiroshima University Hospital, and 6 patients with intraocular tumors were recruited from those treated in the Department of Ophthalmology of the hospital.

\section{MR Imaging Examination}

MR imaging was performed at 1.5T (Signa Excite HD 1.5T; GE Healthcare, Milwaukee, Wisconsin) by using a 76-mm-diameter circular surface receiver coil (GE Healthcare).

The half-Fourier single-shot RARE sequence $(\mathrm{TR} / \mathrm{TE}=$ $1508 / 80 \mathrm{~ms}$, flip angle $=90^{\circ}$, in-plane pixel size $=0.098 \times 0.098$ $\mathrm{mm}$, matrix $=512 \times 512$ with zero-filling interpolation, $\mathrm{FOV}=$ $50 \mathrm{~mm}$, section thickness $=2.1 \mathrm{~mm}$, number of acquisitions $=1$, section acquisition time $=2$ seconds) and the FSPGR sequence $\left(\mathrm{TR} / \mathrm{TE}=30.1 / 2.7 \mathrm{~ms}\right.$, flip angle $=30^{\circ}$, in-plane pixel size $=$ $0.31 \times 0.63 \mathrm{~mm}$, matrix $=256 \times 128, \mathrm{FOV}=80 \mathrm{~mm}$, section thickness $=2.1 \mathrm{~mm}$, number of acquisitions $=1$, and section acquisition time $=4$ seconds) were used in the present study.

For the phantom study, the retinal detachment phantom was fixed in a water bath, and sequential contiguous cross-sectional half-Fourier single-shot RARE and FSPGR images of the phantom were obtained. For the human study, participants were imaged in the supine position; the surface coil was placed in front of their eyes and fixed with tape. Sequential contiguous axial RARE images of the right eye of each volunteer subject were obtained twice in 1 week to assess the imaging, intraobserver, and interobserver reproducibility of RARE volumetry. In the 6 patients with intraocular tumors, sequential contiguous RARE images of the diseased eyes were obtained at least twice during conservative treatment or before and after chemotherapy. In 4 of the 6 patients, fat-saturated FSPGR images before and after the intravenous injection of Gd-DTPA $(0.1 \mathrm{mmol} / \mathrm{kg})$ were also obtained. Each subject (except for a 2-year-old boy under sedation) was asked to stare at a target painted on the inner wall of the magnet gantry, and 1 axial image of the eyeball was acquired; then, the subject was instructed to blink. The same process was repeated to obtain multiple axial images covering the whole eyeball. If an axial image contained motion artifacts, the axial scan was repeated until a still image without artifacts was obtained.

\section{Phantom Data Evaluation}

A total of $1.00,1.50,2.00,2.50,3.00,3.50$, or $4.00 \mathrm{~mL}$ of gadolinium solution was injected into the simulated subretinal space. Then, 2 radiologists (K.T., observer A and N.T., observer B), each with 15 years of experience in clinical MR imaging, manually measured the subretinal area on each sectional image by using manufacturer-supplied software (Advantage Windows 4.2; GE Healthcare). The subretinal fluid volume was calculated by multiplying the sum of the measured subretinal area on each sectional image by each section thickness $(2.1 \mathrm{~mm})$. The measurement error rate was defined as

(measured volume - injected volume)/(injected volume).

Also, SNRs were calculated from the mean signal in a region of interest within the water around the phantom and the SD of the signal of background air on RARE and FSPGR images.

AJNR Am J Neuroradiol 34:870-76 Apr 2013 www.ajnr.org 


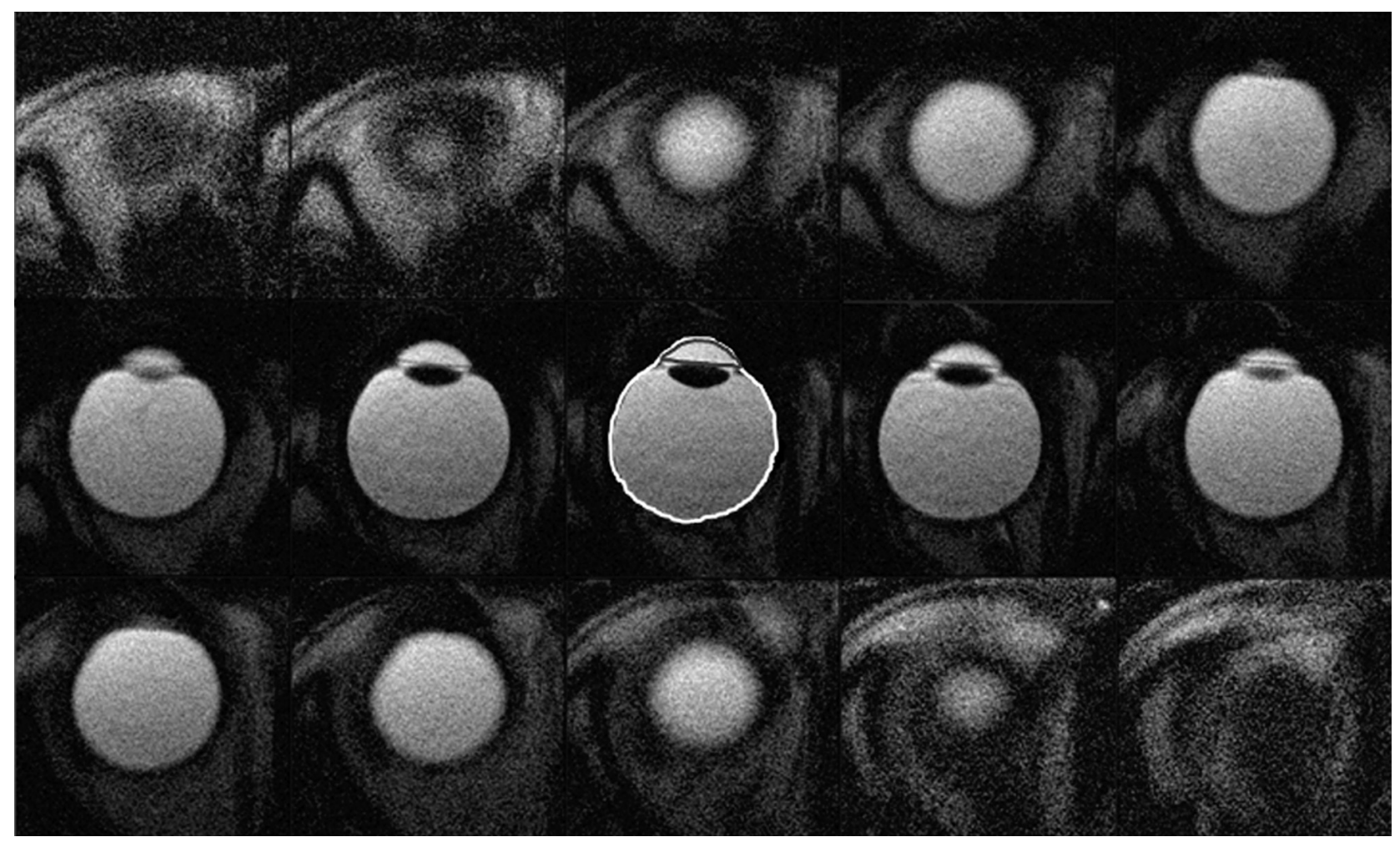

FIG 2. Sequential contiguous half-Fourier single-shot RARE images of the right eye of a female volunteer. Two radiologists manually measured the areas of the anterior chamber and the whole eyeball (enclosed with a gray and a white freehand line in the centered figure, respectively) on each image and calculated their volumes by multiplying the sum of the areas by each section thickness.

Results of the volumetric phantom study ${ }^{\mathrm{a}}$

\begin{tabular}{lccccc}
\hline \multirow{2}{*}{$\begin{array}{c}\text { Infusion } \\
\text { Volume } \\
\left(\times 10^{3} \mathrm{~mm}^{3}\right)\end{array}$} & \multicolumn{3}{c}{ Measured Volume } & $\left(\times 10^{3} \mathrm{~mm}^{3}\right)$ (Error Rate) \\
\cline { 2 - 3 } \cline { 5 - 6 } \cline { 5 - 6 } \cline { 5 - 6 } & \multicolumn{2}{c}{ Observer 1 } & & \multicolumn{2}{c}{ Observer 2} \\
\hline 1.00 & $1.17(0.170)$ & $1.08(0.080)$ & & $1.13(0.130)$ & $1.07(0.070)$ \\
1.50 & $1.64(0.093)$ & $1.61(0.067)$ & & $1.59(0.060)$ & $1.57(0.047)$ \\
2.00 & $2.14(0.070)$ & $2.02(0.010)$ & $2.16(0.080)$ & $2.09(0.045)$ \\
2.50 & $2.74(0.080)$ & $2.60(0.040)$ & & $2.73(0.092)$ & $2.47(-0.012)$ \\
3.00 & $3.15(0.050)$ & $3.06(0.020)$ & & $3.16(0.053)$ & $3.08(0.027)$ \\
3.50 & $3.74(0.069)$ & $3.54(0.011)$ & $3.69(0.054)$ & $3.44(0.017)$ \\
4.00 & $4.10(0.025)$ & $4.05(0.013)$ & $4.17(0.043)$ & $3.98(-0.025)$ \\
\hline
\end{tabular}

a Volumes ranging from 1.00 to $4.00 \mathrm{~mL}$ were injected into the subretinal space. For volumetry using FSPGR on the phantom with $1.00 \mathrm{~mL}$ of subretinal fluid, the measurement error rates of each of the 2 observers were $>0.10$. For other phantom volumetry using FSPGR and all phantom volumetry using half-Fourier single-shot RARE, measurement error rates were $<0.10$. For both observers, all error rates in the measurement of subretinal volumes were smaller on half-Fourier single-shot RARE images than on FSPGR images.

\section{Imaging, Intraobserver, and Interobserver Reproducibility with Human Ocular Volumetry}

To assess imaging, intraobserver, and interobserver reproducibility, each volunteer was scanned twice during 1 week, and the 2 volumetric measurements in each subject were performed individually and blindly by 2 radiologists. When manually measuring the volume of the anterior chamber and the whole eyeball on each scan, ROIs were drawn along the interface between the aqueous (or vitreous) humor and the surrounding soft-tissue structures on RARE images.

\section{Statistical Analysis}

In the phantom and the volunteer studies, statistical analyses were performed with MedCalc for Windows (Version 12.1.0; MedCalc
Software, Mariakerke, Belgium). A paired $t$ test was used to compare the difference in measurement error rates between the 2 sequences in the phantom volumetric study. Bland-Altman analysis, Pearson correlation, and the paired $t$ test were performed to assess the imaging and intraobserver reproducibility of RARE ocular volumetry in the volunteer study. Also, we tested interobserver differences with the $\mathrm{R}$ statistics package (www.r-project.org), and estimated the differences, taking into account the imaging and intraobserver reproducibility. ANOVA was conducted under a null hypothesis that there was no variation between individuals. A value of $P<.01$ represented statistical significance.

\section{Patient Data Evaluation}

Six patients with intraocular tumors ( 2 with choroidal hemangioma undergoing conservative treatment, 2 with suspected malignant choroidal melanoma undergoing conservative treatment, 1 with malignant choroidal melanoma after ruthenium plaque radiation therapy, and 1 with retinoblastoma undergoing chemotherapy) underwent at least 1 follow-up MR imaging examination during at least 6 weeks. An ophthalmologist (T.S.) and a radiologist (K.T.) evaluated the morphologic features of intraocular tumors. Also, the radiologist performed follow-up volumetric analysis by measuring the volumes of whole eyeballs, intraocular tumors, and subretinal fluid (in cases with complicated retinal detachment) in each subject.

\section{RESULTS}

\section{Phantom Study}

Figure $1 B,-C$ shows representative half-Fourier single-shot RARE and FSPGR images of the retinal detachment phantom. Volumet- 

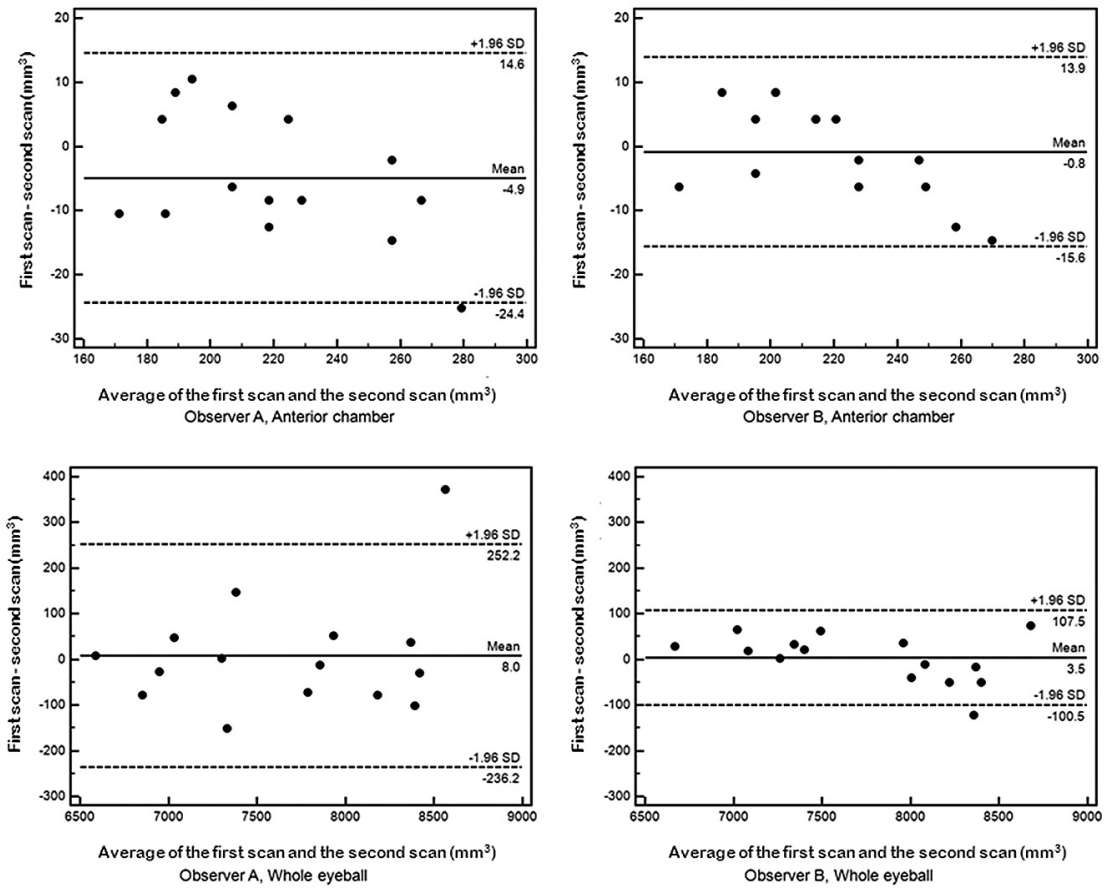

FIG 3. Imaging and intraobserver reproducibility on ocular MR volumetry. The right eyes of 15 volunteers were scanned twice in 1 week. Bland-Altman analysis shows the comparison of the volumes of the anterior chamber or whole eyeball between the first and second scans. Data are shown for each of the 2 observers. The central line (mean) indicates the bias, and the outer broken lines (mean $\pm 2 \mathrm{SDs}$ ) indicate the limits of agreement.
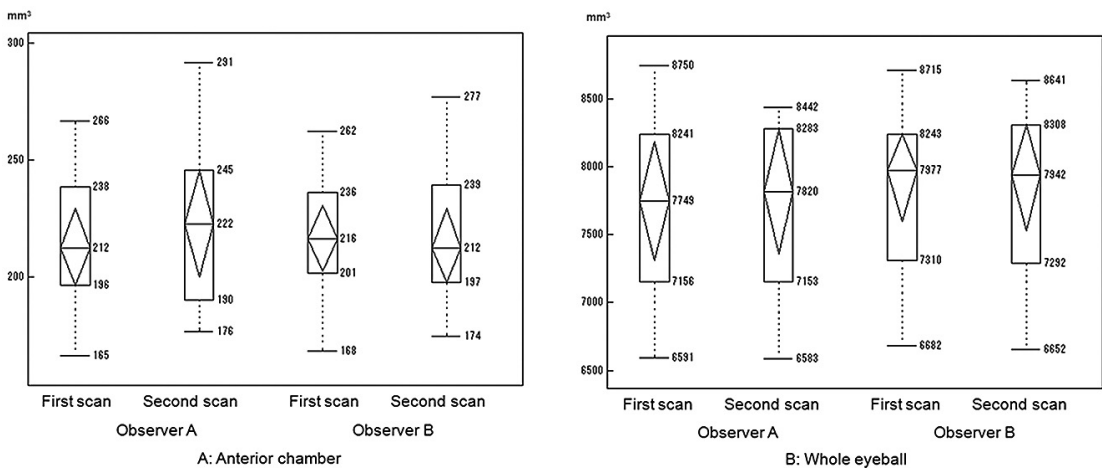

FIG 4. Boxplots showing volumetric data measured by each of the 2 observers in the first and second scans ( $A$, anterior chamber. $B$, whole eyeball). Although no interobserver difference for the measured anterior chamber volumes was evident, volumes of the whole eyeball measured by observer $\mathrm{B}$ were higher than those measured by observer $\mathrm{A}$.

ric data obtained from the phantom with different injection volumes $(1.00-4.00 \mathrm{~mL})$ are listed in the Table. The measurement error rates on RARE volumetry were significantly lower than those on FSPGR volumetry (each observer, $P<.01$ ). SNRs of the RARE images were approximately 2 times higher than those of FSPGR images.

\section{Volunteer Study}

A total of 12-15 contiguous axial images covering the whole eyeball of healthy volunteers were obtained. Fifteen subjects were scanned twice; a total of 30 scans were obtained. On the first attempt with 16 scans, all axial images were obtained without motion artifacts. On the first attempt with 8 scans, there was 1 image with motion artifacts during each scan. On the first attempt with 5 scans, there were 2 images with motion artifacts during each scan. On the first attempt with $1 \mathrm{scan}$, there were 4 images with motion artifacts. In subjects with images with motion artifacts, the axial images were rescanned; and sequential, contiguous, still ocular images were obtained in all subjects. Figure 2 presents sequential contiguous halfFourier single-shot RARE ocular images of a volunteer subject. Approximately 15 minutes per subject was spent drawing ROIs and calculating volumes of the anterior chamber and the whole eyeball.

Bland-Altman plots demonstrate the imaging and intraobserver reproducibility for the anterior chamber and whole-eyeball volumes obtained by each of the 2 observers (Fig 3). In these plots, most of the values were within the range of mean \pm 2 SDs. Pearson correlation coefficients of the imaging and intraobserver reproducibility for the volumes of the anterior chamber and the whole eyeball of observer A were 0.97 and 0.98 , respectively (each, $P<$ .01 ), and those of observer B were 0.98 and 0.99 , respectively (each, $P<.01$ ). Figure 4 shows the interobserver reproducibility of ocular volumetry by using the RARE images. Although there were no interobserver differences in the measured anterior chamber volume $(P=$ .33 ), there was a significant difference between the 2 observers' measurement results of whole-eyeball volume $(P<$ $.01)$. Interobserver differences for the whole-eyeball volume resulted from the large interobserver difference seen for region-of-interest areas at the upper and lower edges of the eyeball, which were significantly higher than at the center of the eyeball $(P<.01)$.

\section{Patient Study}

In all 6 patients with intraocular tumors, at least 1 follow-up ocular volumetry was successfully performed by using sequential contiguous RARE images, and volume changes were evaluated. In 4 of the 6 patients, fat-saturated FSPGR ocular images were obtained before and after intravenous injection of Gd-DTPA. Total study time per patient (including RARE, nonenhanced, and Gdenhanced fat-saturated FSPGR imaging) did not exceed 30 minutes. Although all subjects (except for a 2-year-old boy) were asked to stare at a target for 3-5 seconds for each section scan, no 

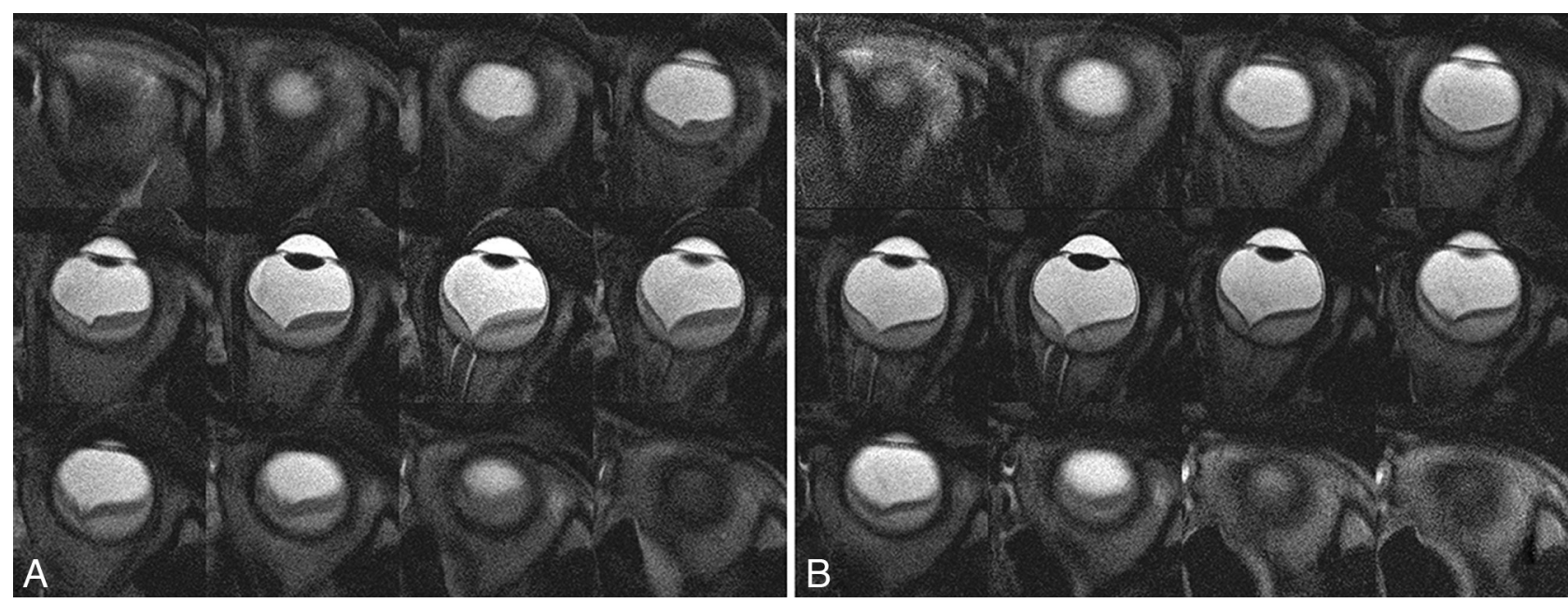

FIG 5. Half-Fourier single-shot RARE images in the follow-up of a 7-year-old girl with Sturge-Weber syndrome. In the first $(A)$ and the second (B) scans, contiguous half-Fourier single-shot RARE images show the convex-shaped relatively low-signal-intensity subretinal fluid and relatively high-signal-intensity choroidal hemangioma in the left eye. The measured volumes of the whole eyeball, the tumor, and the subretinal fluid in the first scan were $6.55 \times 10^{3} \mathrm{~mm}^{3}, 1.32 \times 10^{3} \mathrm{~mm}^{3}$, and $0.61 \times 10^{3} \mathrm{~mm}^{3}$, respectively. The corresponding volumes in the second scan were $6.50 \times$ $10^{3} \mathrm{~mm}^{3}, 1.33 \times 10^{3} \mathrm{~mm}^{3}$, and $0.34 \times 10^{3} \mathrm{~mm}^{3}$, respectively. Although no notable change in the volumes of the whole eyeball and the tumor were found, the volume of subretinal fluid decreased by $44 \%$ after 11 weeks.

subjects complained of eyestrain due to the staring. The volumes of the 5 tumors did not change, whereas the volume of the retinoblastoma under chemotherapy decreased by $71 \%$ (from $3.1 \times$ $10^{2} \mathrm{~mm}^{3}$ to $0.9 \times 10^{2} \mathrm{~mm}^{3}$ ) after 46 days. The volume of subretinal fluid in a patient with choroidal hemangioma associated with Sturge-Weber syndrome decreased by $44 \%$ (from $6.1 \times 10^{2} \mathrm{~mm}^{3}$ to $3.4 \times 10^{2} \mathrm{~mm}^{3}$ ) after 78 days. FSPGR images after Gd-DTPA administration were useful for differentiating tumors from subretinal hemorrhage. Two representative cases are presented in Figs 5 and 6 .

\section{DISCUSSION}

In the present study, half-Fourier single-shot RARE images produced ocular images with higher temporal and spatial resolution than FSPGR images. The phantom study demonstrated that measurement error rates in volumetry by using RARE were lower than those by using FSPGR. Hence, half-Fourier single-shot RARE imaging was chosen for ocular volumetry in a group of healthy volunteers and patients with intraocular tumors. The present volunteer study suggests that ocular volumetry has excellent imaging and intraobserver reproducibility from analysis of sequential contiguous half-Fourier single-shot RARE images. In all patients with intraocular tumors, RARE volumetry was useful for determining changes in tumor volume. On the basis of these results, it is suggested that ocular MR volumetry by using the RARE sequence, a small surface coil, and fixation of the subject's visual focus is both feasible and clinically useful.

Volumetric evaluation can help monitor disease progression and assess therapeutic effects. Although precise ocular volumetric data cannot be obtained with widely used ophthalmic imaging methods such as sonography and OCT, ${ }^{22}$ the present volumetric method by using ultrafast RARE high-resolution MR imaging and visual fixation might be helpful in the follow-up of intraocular lesions.

The scanning times for 1 section in half-Fourier single-shot RARE and fat-saturated FSPGR imaging optimized in this study were 2 seconds and 4 seconds, respectively. Although ocular imaging presents challenges due to voluntary and involuntary eye movements, these fast MR images were useful for obtaining ocular images without motion artifacts. ${ }^{13-16}$ With MR imaging systems with higher gradient strengths and slew rates, T1- and T2weighted ocular imaging with higher SNR and better temporal resolution could be obtained. ${ }^{18,23}$ A 76-mm-diameter commercial surface coil was used, and high spatial resolution images of the eyeball were obtained. However, smaller diameter microscopy surface coils with high sensitivity could be useful for clearer delineation of the eyeball. ${ }^{13,14,17,19}$

Acquiring each axial image of the eyeball while having the subject stare at a target is useful for obtaining multiple axial images of the eyeball with the same visual direction. ${ }^{17}$ When a diseased eye with severe low visual acuity was scanned, the subject was asked to stare at the target through the eye with better visual acuity. In this way, still images of the diseased eye were obtained because both eyes were still during the conjugate gaze.

FSPGR imaging has lower temporal and spatial resolution than RARE imaging, and fat-saturated FSPGR imaging provides poor contrast images of the eyeball. Nonetheless, nonenhanced and Gd-enhanced fat-saturated FSPGR images obtained during visual fixation were useful for evaluating the vascularity of intraocular tumors and for differentiating the tumor from subretinal hemorrhage. If there is insufficient contrast between a tumor and the surrounding normal tissue on RARE images, then Gd-enhanced fat-saturated FSPGR imaging can be used to clarify the tumor spread. ${ }^{24}$

Although excellent imaging and intraobserver reproducibility were found in the volunteer study, interobserver agreement for volumetric data of the whole eyeball was low on RARE volumetry. The interobserver difference was caused by the different ROIs the 2 observers placed on the edge of the eyeball, with the resulting partial volume averaging with the surrounding soft tissue. Volumetric analysis repeated by the same observer in follow-up of 


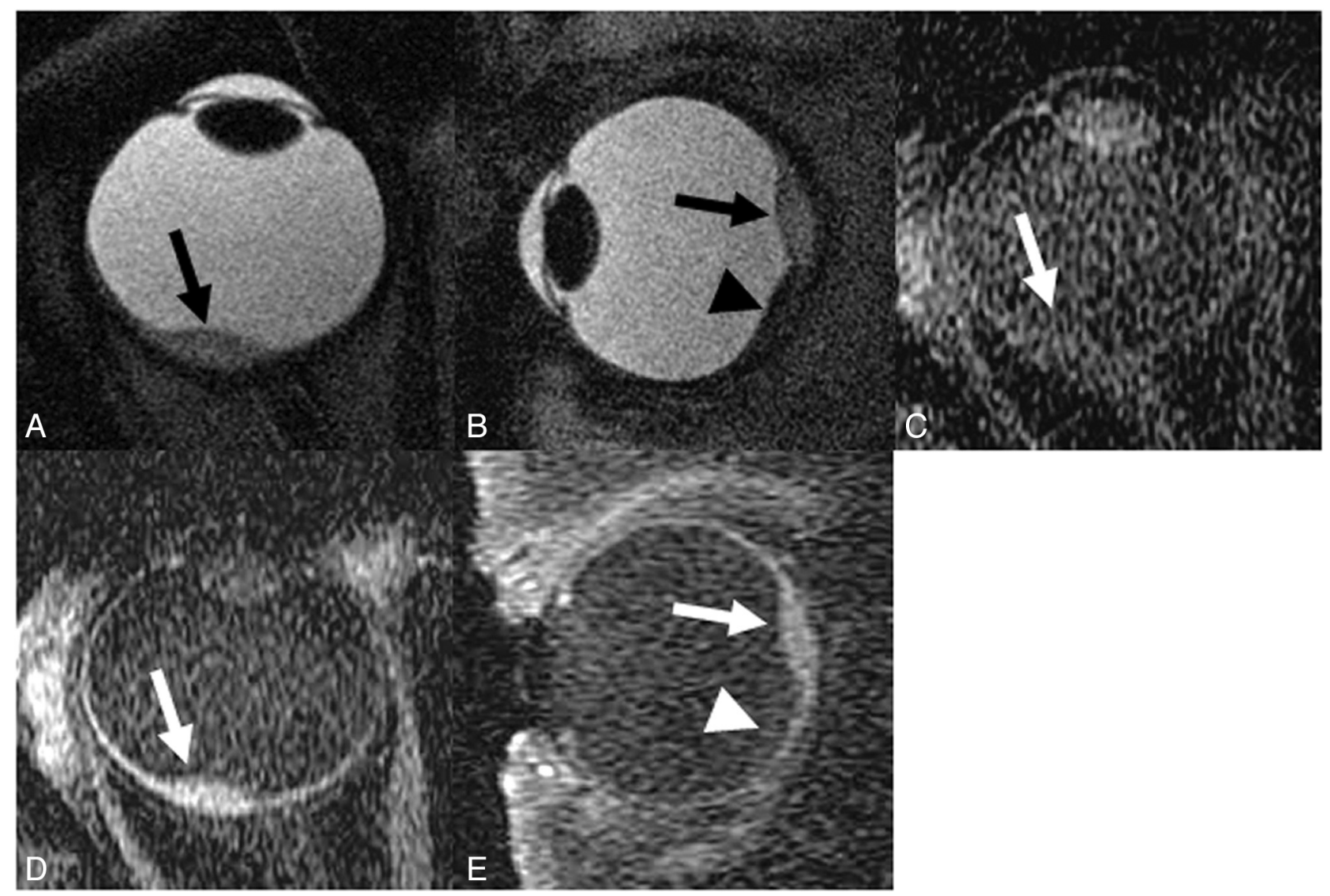

FIG 6. Ocular MR images of a 40-year-old woman with spindle-shaped choroidal hemangioma (arrows) with exudative subretinal fluid (arrowheads). Axial ( $A$ ) and sagittal ( $B$ ) half-Fourier single-shot RARE images show an intermediate-signal-intensity tumor and a low-signal-intensity fluid in the posterior wall of her right eye. Axial fat-saturated FSPGR image $(C)$ shows a low-signal-intensity tumor in the posterior wall of the eye. Axial $(D)$ and sagittal $(E)$ Gd-enhanced fat-saturated FSPGR images show the homogeneous-enhanced tumor and nonenhanced subretinal fluid.

individual patients must be more precise, and an automatic region-of-interest-setting method is needed for ocular volumetry in the future.

The present study has several limitations. First, the half-Fourier single-shot RARE sequence provides heavily $\mathrm{T} 2$-weighted images with low soft-tissue contrast, and the fat-saturated FSPGR sequence with short acquisition time and a small FOV provides low-signal-intensity images of the eyeball. Although it is difficult to differentially diagnose intraocular lesions on the basis of their signal intensity on fast RARE and fat-saturated FSPGR images, RARE and Gd-enhanced fat-saturated FSPGR imaging were useful for evaluating their morphologic features and vascularity, ${ }^{24}$ respectively. Second, the 1.5T MR imaging scanner and the 76$\mathrm{mm}$-diameter insulated commercial receiver coil used in this study are FDA-approved noninvasive imaging tools. In further studies of ocular MR imaging by using equipment with higher gradient strengths and better performing surface receiver coils, the risk of electrical burns, tissue heating, and peripheral nerve stimulation must be considered. ${ }^{25,26}$ Finally, ocular MR imaging is more complex and more expensive than sonography, but fast high-resolution ocular MR imaging with fixing of the subject's visual focus can provide additional information (ie, accurate morphologic and volumetric evaluation of intraocular lesions) that may affect treatment decisions in clinical ophthalmology.

\section{CONCLUSIONS}

Half-Fourier single-shot RARE MR imaging by using a small surface coil while having the subject stare at a target provides sequential contiguous high-quality images of the eyeball. Ocular volumetry from multiple sectional RARE images is feasible and potentially useful for assessment and follow-up of patients with intraocular lesions.

Disclosures: Kazuo Awai-UNRELATED: Consultancy: Daichi-Sankyo (research consultant), Grants/Grants Pending: Toshiba Medical Systems, ${ }^{\star}$ Hitachi Medical Corporation, ${ }^{*}$ Bayer Pharmaceutical, ${ }^{*}$ Comments: research grants. ${ }^{\star}$ Money paid to the institution.

\section{REFERENCES}

1. Ozdemir H, Yucel C, Aytekin C, et al. Intraocular tumors: the value of spectral and color Doppler sonography. Clin Imaging 1997;21:77-81

2. Fielding JA. The assessment of ocular injury by ultrasound. Clin Radiol 2004;59:301-12

3. Weisbrod DJ, Pavlin CJ, Emara K, et al. Small ciliary body tumors: ultrasound biomicroscopic assessment and follow-up of 42 patients. Am J Ophthalmol 2006;141:622-28

4. Costa RA, Skaf M, Melo LAS, et al. Retinal assessment using optical coherence tomography. Prog Retin Eye Res 2006;25:325-53

5. Srinivasan VJ, Wojtkowski M, Witkin AJ, et al. High-definition and 3-dimensional imaging of macular pathologies with high-speed ul-

AJNR Am J Neuroradiol 34:870-76 Apr 2013 www.ajnr.org

875 
trahigh-resolution optical coherence tomography. Ophthalmology 2006;113:2054.e1-14

6. Radhakrishnan S, Goldsmith J, Huang D, et al. Comparison of optical coherence tomography and ultrasound biomicroscopy for detection of narrow anterior chamber angles. Arch Ophthalmol 2005; 123:1053-59

7. Lakits A, Prokesch R, Scholda C, et al. Orbital helical computed tomography in the diagnosis and management of eye trauma. Ophthalmology 1999;106:2330-35

8. Joseph DP, Pieramici DJ, Beauchamp NJ Jr. Computed tomography in the diagnosis and prognosis of open-globe injuries. Ophthalmology 2000;107:1899-906

9. Galluzzi P, Hadjistilianou T, Cerase A, et al. Is CT still useful in the study protocol of retinoblastoma? AJNR Am J Neuroradiol 2009;30:1760-65

10. McCaffery S, Simon EM, Fischbein NJ, et al. Three-dimensional high-resolution magnetic resonance imaging of ocular and orbital malignancies. Arch Ophthalmol 2002;120:747-54

11. Simon EM, McCaffery S, Rowley HA, et al. High-resolution 3D T2weighted fast spin echo: new applications in the orbit. Neuroradiology 2003;45:489-92

12. Daftari IK, Aghaian E, O'Brien JM, et al. 3D MRI-based tumor delineation of ocular melanoma and its comparison with conventional techniques. Med Phys 2005;32:3355-62

13. Tanitame K, Sasaki K, Sone T, et al. Anterior chamber configuration in patients with glaucoma: MR gonioscopy evaluation with halfFourier single-shot RARE sequence and microscopy coil. Radiology 2008;249:294-300

14. Tanitame K, Sasaki K, Sone T, et al. Optimal fast T2-weighted magnetic resonance microscopy imaging of the eye and its clinical application. J Magn Reson Imaging 2010;31:1210-14

15. Chung HW, Chen CY, Zimmerman RA, et al. T2-weighted fast MR imaging with true FISP versus HASTE: Comparative efficacy in the evaluation of normal fetal brain maturation. AJR Am J Roentgenol 2000;175:1375-80

16. Zand KR, Reinhold C, Haider MA, et al. Artifacts and pitfalls in MR imaging of the pelvis. J Magn Reson Imaging 2007;26:480-97

17. Obata $\mathrm{T}$, Uemura $\mathrm{K}$, Nonaka $\mathrm{H}$, et al. Optimizing T2-weighted magnetic resonance sequences for surface coil microimaging of the eye with regard to lid, eyeball and head moving artifacts. Magn Reson Imaging 2006;24:97-101

18. Lemke AJ, Alai-Omid M, Hengst SA, et al. Eye imaging with a 3.0-T MRI using a surface coil-a study on volunteers and initial patients with uveal melanoma. Eur Radiol 2006;16:1084-89

19. Georgouli T, Chang B, Nelson M, et al. Re: "use of high resolution microscopy coil MRI for depicting orbital anatomy": ORBIT issue 27.2 (page 107-114) - answer to reader comments. Orbit 2009;28:208

20. Lemke AJ, Kazi I, Mergner U, et al. Retinoblastoma: MR appearance using a surface coil in comparison with histopathological results. Eur Radiol 2007;17:49-60

21. de Graaf P, Knol DL, Moll AC, et al. Eye size in retinoblastoma: MR imaging measurements in normal and affected eyes. Radiology 2007;244:273-80

22. Finger PT, Romero JM, Rosen RB, et al. Three-dimensional ultrasonography of choroidal melanoma: localization of radioactive eye plaques. Arch Ophthalmol 1998;116:305-12

23. Lee VS, Hecht EM, Taouli B, et al. Body and cardiovascular MR imaging at 3.0 T. Radiology 2007;244:692-705

24. Adam G, Brab M, Bohndorf K, et al. Gadolinium-DTPA-enhanced MRI of intraocular tumors. Magn Reson Imaging 1990;8:683-89

25. Kuhl CK, Traber F, Schild HH. Whole-body high-field-strength (3.0-T) MR imaging in clinical practice. Part I. Technical considerations and clinical applications. Radiology 2008;246:675-96

26. Kuhl CK, Traber F, Gieseke J, et al. Whole-body high-field-strength (3.0-T) MR imaging in clinical practice. Part II. Technical considerations and clinical applications. Radiology 2008;247:16-35 